\title{
European Journal of Preventive Cardiology \\ http://cpr.sagepub.com/
}

\section{Inside ventilatory regulation in pulmonary hypertension: several hidden data are still undiscovered \\ Piergiuseppe Agostoni, Stefania Farina, Anna Apostolo and Susanna Sciomer}

European Journal of Preventive Cardiology 2014 21: 268 originally published online 17 July 2013

DOI: $10.1177 / 2047487313497866$

The online version of this article can be found at:

http://cpr.sagepub.com/content/21/3/268



European Association for Cardiovascular Prevention and Rehabilitation

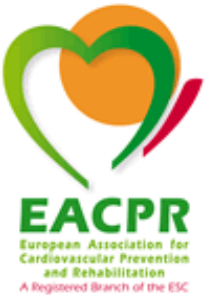

Additional services and information for European Journal of Preventive Cardiology can be found at:

Email Alerts: http://cpr.sagepub.com/cgi/alerts

Subscriptions: http://cpr.sagepub.com/subscriptions

Reprints: http://www.sagepub.com/journalsReprints.nav

Permissions: http://www.sagepub.com/journalsPermissions.nav

>> Version of Record - Feb 19, 2014

OnlineFirst Version of Record - Jul 17, 2013

What is This? 


\section{Inside ventilatory regulation in pulmonary hypertension: several hidden data are still undiscovered}

\author{
Piergiuseppe Agostoni ${ }^{1,2,3}$, Stefania Farina', Anna Apostolo' \\ and Susanna Sciomer ${ }^{4}$
}

Cardiology

20I4, Vol. 2I(3) 268-27|

(C) The European Society of

Cardiology 2013

Reprints and permissions:

sagepub.co.uk/journalsPermissions.nav DOI: I0.1 I77/20474873 |3497866 ejpc.sagepub.com

(S)AGE

\begin{abstract}
During exercise, ventilation for a given work rate is increased in pulmonary hypertension. In the present editorial a new look at the ventilatory behaviour, which may change the common approach to $\mathrm{VE} / \mathrm{VCO}_{2}$ relationship analysis, is presented.
\end{abstract}

The behaviour of ventilation (VE) in a ramp protocol exercise test is peculiar, and it implies relevant physiological information. In a healthy individual, VE increase during exercise is characterized by four linear phases, during which the steepness of the VE vs. workload relationship progressively increases (Figure 1). The first phase is delimited by the beginning of loaded pedalling (provided that an appropriate unloaded exercise period has been performed) and the anaerobic threshold; the second phase is between anaerobic threshold and the respiratory compensation point; the third between the respiratory compensation point and peak exercise; and the fourth phase, usually very brief, is sometimes observed in fit individuals close to peak exercise and is characterized by an extremely elevated slope of the VE vs. workload relationship.

Why does the VE relationship have this peculiar behaviour? The classical physiological interpretation is a switch in $\mathrm{VE}$ driver during exercise, being $\mathrm{VO}_{2}$ at the beginning of exercise, $\mathrm{VCO}_{2}$ during the isocapnic buffering period (between anaerobic threshold and the respiratory compensation point), unbuffered acidosis above the respiratory compensation point, and heat exchange at peak exercise in some fit individual. The last one is most evident in fur-coated animals, in which heat cannot or can minimally be eliminated by sweating, and ventilation becomes the main heat exchanger during exercise through the mechanism of panting. Indeed, at the beginning of exercise, below the anaerobic threshold, energy production is aerobic, so that $\mathrm{VO}_{2}$ drives VE, and above the anaerobic threshold energy production is both aerobic and anaerobic.
Anaerobic energy production implies an extra $\mathrm{CO}_{2}$ production to buffer acidosis, which is compensated up to the respiratory compensation point and not compensated above it. Accordingly, $\mathrm{VCO}_{2}$ and unbuffered acidosis drive $\mathrm{VE}$ in the isocapnic buffering period and above the respiratory compensation point, respectively. ${ }^{1}$

The behaviour of the $\mathrm{VCO}_{2}$ vs. workload relationship is different, being linear but characterized by two instead of four linear phases, with a steeper slope in the second phase (Figure 2). The first phase is from the beginning of exercise to the anaerobic threshold; the second is from anaerobic threshold to peak exercise. Indeed, above the anaerobic threshold, more $\mathrm{CO}_{2}$ is needed to buffer lactic acid. The $\mathrm{VE}$ vs. $\mathrm{VCO}_{2}$ relationship also has two phases; the first from the beginning of exercise to the respiratory compensation point; and the second above the respiratory compensation point (Figure 3). Indeed, up to the respiratory compensation

\footnotetext{
'Centro Cardiologico Monzino, IRCCS, Milan, Italy

${ }^{2}$ Department of Clinical Sciences and Community Health, Cardiovascular Section, University of Milano, Milan, Italy

${ }^{3}$ Division of Pulmonary and Critical Care and Medicine, Department of Medicine, University of Washington, Seattle, WA, USA

${ }^{4}$ Department of Cardiovascular and Respiratory Sciences, "La Sapienza" University, Rome, Italy
}

\section{Corresponding author:}

Piergiuseppe Agostoni, Centro Cardiologico Monzino, IRCCS,

Dipartimento di Scienze Cliniche e Medicina di Comunità, Università degli Studi di Milano, via Parea 4, 20I38, Milano, USA.

Email: piergiuseppe.agostoni@unimi.it 


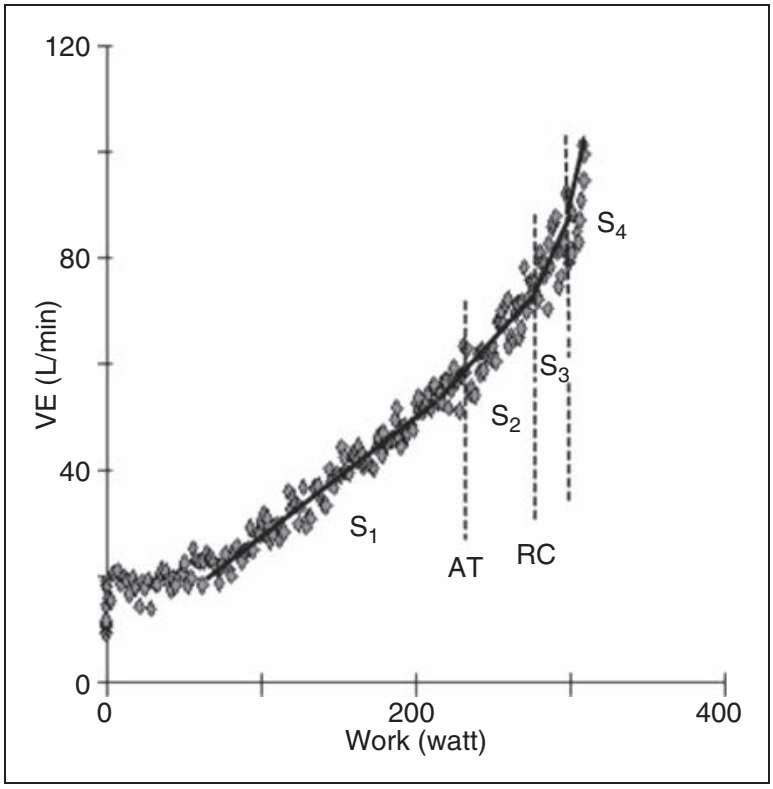

Figure I. Ventilation (VE) vs. workload relationship in an healthy individual.

VE increase during exercise is characterized by four linear phases characterized by a progressively increasing slope: $\left(\mathrm{S}_{\mathrm{I}}\right)$ delimited by the beginning of VE increase (usually at the beginning of loaded pedaling) and the anaerobic threshold; $\left(S_{2}\right)$ between anaerobic threshold and the respiratory compensation point; $\left(S_{3}\right)$ between the respiratory compensation point and peak exercise; and $\left(S_{4}\right)$ usually very brief, is observed some times in fit individuals. AT, anaerobic threshold; RC, respiratory compensation point.

point, $\mathrm{VE}$ and $\mathrm{VCO}_{2}$ increases are parallel, so that the slope of the relationship between the two is constant. The presence and the length of the isocapnic buffering period (i.e. the time lag between the anaerobic threshold and the respiratory compensation point) are related to the intensity of the workload increase and to the amount of $\mathrm{CO}_{2}$ available for lactic acid buffering. The amount of $\mathrm{CO}_{2}$ is related to the amount of tissue available for $\mathrm{CO}_{2}$ storing, to pre-exercise ventilation and to ventilation at the beginning of exercise. At high altitude, for instance, where hypoxia drives VE and hyperventilation is a constant, the isocapnic buffering period disappears or it is significantly reduced. , $^{2,3}$

Plenty of studies have reported the VE vs. $\mathrm{VCO}_{2}$ behaviour as pivotal to define the efficiency/inefficiency of ventilation and prognosis in several pathological conditions including heart failure and pulmonary hypertension. ${ }^{4-7}$ But how should we measure the relationship between $\mathrm{VE}$ and $\mathrm{VCO}_{2}$ ? Some authors prefer to analyse the ratio of $\mathrm{VE} / \mathrm{VCO}_{2}$ and to avoid the analysis of the slope of the $\mathrm{VE}$ vs. $\mathrm{VCO}_{2}$ relationship. Indeed, it is much simpler to calculate the ratio, either at peak exercise or at the anaerobic threshold, or just to

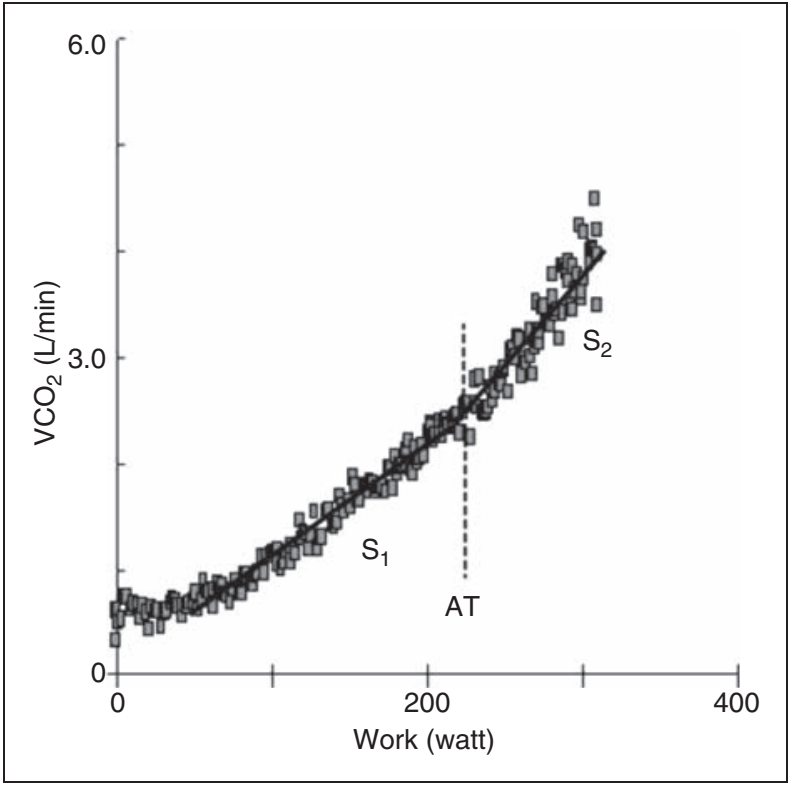

Figure 2. $\mathrm{VCO}_{2}$ vs. workload relationship in a healthy individual.

The relationship is characterized by two instead of four linear phases, with a higher slope in the second: $\left(\mathrm{S}_{\mathrm{I}}\right)$ from the beginning of exercise to the anaerobic threshold; and $\left(\mathrm{S}_{2}\right)$ from anaerobic threshold to peak exercise. AT, anaerobic threshold; $\mathrm{VCO}_{2}$, carbon dioxide production.



Figure 3. Ventilation vs. $\mathrm{VCO}_{2}$ relationship in a healthy individual.

Two phases are shown: $\left(S_{1}\right)$ from the beginning of exercise to the respiratory compensation point; and $\left(S_{2}\right)$ above the respiratory compensation point. RC, respiratory compensation point. 
consider the lowest observed during a ramp protocol exercise. ${ }^{8}$ But doing so, the dynamic behaviour of the $\mathrm{VE}$ vs. $\mathrm{VCO}_{2}$ relationship is lost, and, most importantly, the information hidden in the dynamic behaviour of the $\mathrm{VE}$ vs. $\mathrm{VCO}_{2}$ relationship is lost. There is also a dilemma about in which phase of exercise the analysis of the slope of the $\mathrm{VE}$ vs. $\mathrm{VCO}_{2}$ relationship should be done. From a physiological point of view, the answer seems trivial: from the beginning of exercise up to the respiratory compensation point where $\mathrm{VE}$ is driven by $\mathrm{VO}_{2}$ at the beginning and by $\mathrm{VCO}_{2}$ later, following a distinct linear relationship with $\mathrm{VO}_{2}$ and $\mathrm{VCO}_{2}$ increases being parallel. Indeed, what is the possible meaning of the $\mathrm{VE}$ vs. $\mathrm{VCO}_{2}$ relationship when the two are not physiologically linked as during heavy exercise when VE is driven by unbuffered acidosis and, eventually, by the thermal stress of exercise?

However, a previous study of Cohen Solail's group 9 in heart failure patients and the study of Ferreira et al. ${ }^{10}$ in pulmonary hypertension patients reported in the present issue of the European Journal of Preventive Cardiology show that the strongest prognostic information is obtained if the slope of the VE vs. $\mathrm{VCO}_{2}$ relationship is calculated through the entire loaded exercise. Although this finding does not detract from the above-reported physiological principles, this observation suggests analysing the physiology of exercise from a different point of view, considering that patients and not healthy subjects are evaluated.

To do so, we took the liberty to modify Figure 1 of Ferreira et al., ${ }^{10}$ which shows an exemplificative case of $\mathrm{VE}$ vs. $\mathrm{VCO}_{2}$ behaviour in a primitive pulmonary hypertension patient. The lines of the $\mathrm{VE}$ vs. $\mathrm{VCO}_{2}$ relationship were drawn manually from the beginning of exercise to the respiratory compensation point and from the respiratory compensation point to peak exercise (Figure 4). VE is dead space ventilation + alveolar space ventilation. If the $\mathrm{Y}$-intercept of the $\mathrm{VE}$ vs. $\mathrm{VCO}_{2}$ relationship is positive, this means that alveolar ventilation proportionally increases more than dead space ventilation; if the Y-intercept is 0 , then dead space and alveolar ventilation increases during exercise are proportionally identical; if the $\mathrm{Y}$-intercept is negative, this means that dead space ventilation proportionally increases more than alveolar ventilation. This is the case in the example reported by Ferreira et al. for the $\mathrm{VE}$ vs. $\mathrm{VCO}_{2}$ relationship above the respiratory compensation point. ${ }^{10}$

This reasoning, which we recognize has been built on a single case, suggests that when the increase of dead space ventilation is proportionally greater than the increase in alveolar ventilation, the inefficiency of VE is high and prognosis is poor. It may be interesting to find out whether, contrary to what is believed in physiology of exercise, the analysis of $\mathrm{VE}$ vs. $\mathrm{VCO}_{2}$ (or



Figure 4. Modification of Figure I by Ferreira et al. ${ }^{10}$ The dotted line has been drawn from the beginning of loaded exercise to the respiratory compensation point and continuous line from the respiratory compensation point to peak exercise. See text for interpretation. VE, ventilation; $\mathrm{VCO}_{2}$, carbon dioxide production.

work) slope from the respiratory compensation point to peak exercise has a prognostic capability stronger than the analysis of these relationships in any other part of exercise. If, in a ramp protocol exercise, a further alveolar ventilation increase is obtained at the cost of a relevant dead space ventilation increase and if this is associated to poorer prognosis, then this finding suggests analysing the VD/VT behaviour during exercise.

Actually, the present is an old, forgotten idea by John Butler, ${ }^{11}$ whose reappraisal will probably improve our prognostic capability in pulmonary hypertension patients. The paper of Ferreira et al..$^{10}$ is an important step in this direction.

Finally, because hyperventilation is a dominant event in pulmonary hypertension, as at high altitude, it may be interesting to evaluate whether the length, normalized for the workload increase, of the isocapnic buffering period is reduced, being $\mathrm{CO}_{2}$ deposit reduced by hyperventilation, and whether this has prognostic capability. Similarly, a lot of prognostic information may be hidden in the VE/PETCO ${ }_{2}$ (end tidal $\mathrm{CO}_{2}$ pressure) ratio at the anaerobic threshold or at the respiratory compensation point again as an index of hyperventilation. In conclusion, the paper by Ferreira et al. ${ }^{10}$ is like a Pandora's box for ideas about how to analyze the VE behaviour in primitive pulmonary 
hypertension patients and, we congratulate the authors for this study.

\section{Conflict of interest}

The authors declare that there are no conflicts of interest.

\section{Funding}

This research received no specific grant from any funding agency in the public, commercial, or not-for-profit sectors.

\section{References}

1. Wasserman K, Hansen JE, Sue DY, et al. Physiology of exercise. In: (eds) Principles of exercise testing and interpretation, 5th ed. Philadelphia, USA: Lippincott Williams \& Wilkins-Wolters Kluwer Health, 2012, chapter 2, pp.9-61.

2. Agostoni P, Valentini M, Magri D, et al. Disappearance of isocapnic buffering period during increasing work rate exercise at high altitude. Eur J Cardiovasc Prev Rehabil 2008; 15: 354-358.

3. Agostoni P, Contini M, Cattadori G, et al. Lung function with carvedilol and bisoprolol in chronic heart failure: is beta selectivity relevant? Eur J Heart Fail 2007; 9: 827-833.

4. Arena R, Myers J, Abella J, et al. Development of a ventilatory classification system in patients with heart failure. Circulation 2007; 115: 2410-2417.
5. Gitt AK, Wasserman K, Kilkowski C, et al. Exercise anaerobic threshold and ventilatory efficiency identify heart failure patients for high risk of early death. Circulation 2002; 106: 3079-3084.

6. Yasunobu Y, Oudiz RJ, Sun XG, et al. End-tidal $\mathrm{PCO}_{2}$ abnormality and exercise limitation in patients with primary pulmonary hypertension. Chest 2005; 127: 1637-1646.

7. Groepenhoff H, Vonk-Noordegraaf A, Boonstra A, et al. Exercise testing to estimate survival in pulmonary hypertension. Med Sci Sports Exerc 2008; 40: 1725-1732.

8. Sun XG, Hansen JE, Garatachea N, et al. Ventilatory efficiency during exercise in healthy subjects. $\mathrm{Am} \mathrm{J}$ Respir Crit Care Med 2002; 166: 1443-1448.

9. Tabet JY, Beauvais F, Thabut G, et al. A critical appraisal of the prognostic value of the $\mathrm{VE} / \mathrm{VCO}_{2}$ slope in chronic heart failure. Eur J Cardiovasc Prev Rehabil 2003; 10: 267-272.

10. Ferreira EMV, Ota-Arakaki J, Ramos R, et al. Optimizing th evaluation of excessive exercise ventilation for prognosis assessment in pulmonary arterial hypertension. Eur J Prev Cardiol 2013 (Epub ahead of print).

11. Agostoni PG and Butler J. Cardiac evaluation. In: Murray JF and Nadel JA (eds) Textbook of respiratory disease, 2nd edn. Philadelphia: WB Saunders, 1994, pp.943-962. 